\title{
Methylene blue added to a hypertonic-hyperoncotic solution increases short-term survival in experimental cardiac arrest*
}

\author{
Adriana Miclescu, MD, PhD; Samar Basu, PhD; Lars Wiklund, MD, PhD
}

Objective: Methylene blue (MB), a free-radical scavenger inhibiting the production and actions of nitric oxide, may counteract excessive vasodilatation induced by nitric oxide during cardiac arrest. Effects of MB in cardiac arrest and cardiopulmonary resuscitation were investigated.

Design: Randomized, prospective, laboratory animal study.

Setting: University animal research laboratory.

Subjects: A total of 63 piglets of both sexes.

Interventions: A pig model of extended cardiac arrest (12 mins of untreated cardiac arrest and 8 mins of cardiopulmonary resuscitation) was employed to assess the addition or no addition of MB to a hypertonic saline-dextran solution. These two groups (MB and hypertonic saline-dextran group [MB group] and hypertonic saline-dextran-only group) of 21 animals were each compared with a group receiving isotonic saline $(n=21)$.

Measurements and Main Results: Although the groups were similar in baseline values, 4-hr survival in the MB group was increased $(p=.02)$ in comparison with the isotonic saline group. Hemodynamic variables were somewhat improved at 15 mins after restoration of spontaneous circulation in the MB group compared with the other two groups. The jugular bulb levels of 8-isoprostane-prostaglandin $\mathrm{F}_{2 \alpha}$ and 15-keto-dihydro-prostaglandin $F_{2 \alpha}$ (indicators of peroxidation and inflammation) were significantly decreased in the MB group compared with the isotonic saline group. Significant differences were recorded between the three groups in levels of protein S-100 $\beta$ (indicator of neurologic injury), with lower levels in the MB group compared with the isotonic saline and hypertonic saline-dextran-only groups. Troponin I and myocardial muscle creatine kinase isoenzyme arterial concentrations (indicators of myocardial damage) were also significantly lower in the MB group.

Conclusions: MB co-administered with a hypertonic-hyperoncotic solution increased 4-hr survival vs. saline in an experimental porcine model of cardiac arrest and reduced oxidative, inflammatory, myocardial, and neurologic injury. (Crit Care Med 2006; 34:2806-2813)

KeY WoRDS: experimental cardiac arrest; methylene blue; saline hypertonic; circulation; survival; organ injury hort-term outcome after cardiac arrest treated by cardiopulmonary resuscitation $(\mathrm{CPR})$ is dependent on the perfusion pressure and blood flow generated, among other factors. Global ischemia associated with cardiac arrest is an important trigger that activates nitric oxide synthase (NOS), which stimulates nitric oxide (NO) production (1). NO induces vasodilatation through a direct activation of soluble guanylate cyclase in vascular smooth muscle, thereby increasing production of cyclic guanosine monophosphate (cGMP) (2). The excessive NO-mediated va-

${ }^{\star}$ See also p. 2862.

From the Department of Surgical Sciences/ Anesthesiology and Intensive Care (AM, LW) and the Department of Public Health and Caring Sciences/ Geriatrics/Clinical Nutrition (SB), Uppsala University Hospital, Uppsala, Sweden.

The authors have not disclosed any potential conflicts of interest.

Supported, in part, by the Laerdal Foundation for Acute Medicine, Stavanger, Norway.

Copyright (C) 2006 by the Society of Critical Care Medicine and Lippincott Williams \& Wilkins

DOI: 10.1097/01.CCM.0000242517.23324.27 sodilatation that occurs during and immediately after cardiac arrest and during resuscitation may affect the rate of successful resuscitation (3) by reducing the perfusion pressure. Therefore, in the search for adjunctive and alternative methods of increasing cerebral and myocardial perfusion pressure and subsequent blood flow during CPR, administration of a drug that counteracts NOinduced vasodilatation might be warranted.

Methylene blue (MB) counteracts NOmediated vasodilatation by blocking the soluble guanylate cyclase (4) via the oxidation of its active hemo-center (5) or by inactivation of its hemo-deficient apoenzyme (6). Moreover, MB is a potent inhibitor of NOS and binds to and, in addition, inactivates NO (7).

Furthermore, $\mathrm{MB}$ is known to inhibit the formation of free oxygen radicals and superoxides (reactive oxygen species) by competing with molecular oxygen for the transfer of electrons by xanthine oxidase (8), and it is effective in attenuating ischemia-reperfusion syndrome (9). As a kind of free-radical scavenger, MB may offer a therapeutic option in the postresuscitation syndrome because free-radical-mediated injury has been suspected to be one of the major causes underlying postischemic reperfusion injury (10).

To elucidate the effects of MB in CPR, we selected an experimental pig model of extended circulatory arrest. A similar model has been employed to assess the efficacy of hypertonic-hyperoncotic solutions, which have been demonstrated to improve myocardial blood flow during CPR (11) and by acting as a cardioprotective and neuroprotective agent in the reperfusion period (12, 13). Thus, one trial group received a combination of hypertonic saline with dextran and $\mathrm{MB}$, and a second group received hypertonic saline with dextran only. These two groups were compared with a control group that received normal isotonic saline only. The pharmacologic intervention was administered during CPR and during the initial reperfusion period after cardiac arrest. The null hypothesis for the experiments was that there would be no difference in either survival or circulatory or nervous system effects between the control group and the two experimental groups. 


\section{MATERIALS AND METHODS}

The regional review board for animal experimentation in Uppsala, Sweden, approved this prospective, randomized, laboratory animal study.

Animals. A total of 63 Swedish domestic piglets, aged 12-15 wks and with a mean weight of $25.1 \pm 2.1 \mathrm{~kg}$, obtained from a single provider were delivered to the laboratory on the morning of the experiment. The piglets fasted $6 \mathrm{hrs}$ before the experiment but had free access to water.

Anesthesia. The animals received an intramuscular injection of $6 \mathrm{mg} / \mathrm{kg}$ tiletamine and zolazepam (Zoletil, Reading, France) combined with $2.2 \mathrm{mg} / \mathrm{kg}$ xylazine (Rompun, Bayer, Germany) and $0.04 \mathrm{mg} / \mathrm{kg}$ atropine. A peripheral venous catheter (18-gauge) was inserted in an ear vein and was used for induction and maintenance of anesthesia and for fluid administration. All the piglets received an intravenous injection of $20 \mathrm{mg}$ of morphine (Morfin Meda, Solna, Sweden). If needed, 100 mg of ketamine (Ketaminol, Veterinaria AG, Switzerland) was added as an intravenous bolus to achieve surgical anesthesia. Absence of motor response to painful stimuli was considered indicative of adequate anesthesia. An intravenous infusion of $8 \mathrm{mg} \cdot \mathrm{kg}^{-1} \cdot \mathrm{hr}^{-1}$ pentobarbital (Apoteket, Sweden), $0.5 \mathrm{mg} \cdot \mathrm{kg}^{-1} \cdot \mathrm{hr}^{-1}$ morphine, and $0.25 \mathrm{mg} \cdot \mathrm{kg}^{-1} \cdot \mathrm{hr}^{-1}$ pancuronium bromide (Pavulon, Organon, Netherlands) was started and used for maintenance of anesthesia. The piglets were secured in a supine position and were tracheostomized. The animals were mechanically ventilated (Servo $900 \mathrm{C}$, Siemens-Elema, Solna, Sweden) with a 30/70 mixture of oxygen/nitrous oxide during the preparation and $30 \%$ oxygen in air after the preparation, providing an $\mathrm{FIO}_{2}$ of 0.3 . The mechanical ventilation was volume controlled with an inspiratory/expiratory ratio of $1: 2$, a fixed frequency of $25 / \mathrm{min}$, and a minute volume set to maintain arterial $\mathrm{PaCO}_{2}$ at an average of 34-41 mm Hg (4.5-5.5 $\mathrm{kPa})$. A positive end-expiratory pressure of $5 \mathrm{~cm} \mathrm{H}_{2} \mathrm{O}$ was applied. The capnogram and peripheral oxygen saturation were displayed continuously $\left(\mathrm{CO}_{2} \mathrm{SMO}\right.$ Plus-8100, Novametrix, Wallingford, CT), as were leads II and V5 of the electrocardiogram.

Fluid Administration. All animals received fluid replacement with acetated Ringer solution (Ringer-acetat, Fresenius Kabi, Stockholm, Sweden) as follows: $30 \mathrm{~mL} / \mathrm{kg}$ during the first hour of preparation and thereafter a continuous infusion of $10 \mathrm{~mL} \cdot \mathrm{kg}^{-1} \cdot \mathrm{hr}^{-1}$ to obtain a pulmonary artery occlusion pressure of $7-10 \mathrm{~mm} \mathrm{Hg}$.

Surgical Preparation. An 18-gauge arterial catheter was advanced into the aortic arch via a branch of the right external carotid artery for withdrawal of blood samples and measurement of blood pressure. A 14-gauge, salinefilled, double-lumen catheter was placed into the right atrium via a cutdown of the right external jugular vein to measure right atrial pressure and for drug administration. Along with that, a 7-Fr, fluid-filled, pulmonary artery catheter (CritiCath, Ohmeda Medical Devices, Oxnard, CA) was floated into occlusion position in the pulmonary artery and thereafter withdrawn 1-2 cm to measure cardiac output, artery occlusion pressure, and to sample mixed venous blood. These catheters were connected to pressure transducers, which were calibrated to atmospheric pressure at the level of the right atrium. The left internal jugular vein was cannulated in the cephalad direction, and a catheter (18-gauge) was advanced to the jugular bulb. To limit contamination from extracerebral blood, the position of the catheter tip was confirmed by lateral neck fluoroscopy and a contrast injection. A 10 -mm burr hole was made between the middle and coronal sutures of the right hemisphere, and the dura was opened. Through this hole, a laser-Doppler flow probe (MT B500-43, Periflux PF 2B Laser-Doppler Flowmeter, Perimed, Stockholm, Sweden) was placed directly on the surface of the right frontal cortex. The probe was fixed by suturing dura and skin.

Measurements and Samples. Hemodynamic variables including lead II and V5 electrocardiogram recordings, heart rate, systemic arterial blood pressure, right atrial pressure, and pulmonary artery pressure were continuously displayed (Solar 8000 monitor, Marquette Medical Systems, Milwaukee, WI) and recorded (Workbench 3.0, Strawberry Tree, Sunnyvale, CA). Cardiac output was measured as a mean of three measurements, using the thermodilution technique at baseline and repeatedly during the postresuscitation period. Pulmonary artery occlusion pressure was recorded at the same points as cardiac output. Coronary perfusion pressure was calculated as the difference between the diastolic aortic pressure and the simultaneously measured right atrial pressure. Cerebral cortical blood flow was recorded every 5 secs by a computer and presented as a fraction of the steady-state baseline flow level before induction of ventricular fibrillation. Derived variables, including total systemic vascular resistance and left ventricular stroke work, were calculated by standard formulas. Samples of arterial, jugular, and pulmonary arterial blood were taken for blood gas analysis and acid-base balance (ABL 300, Radiometer, Copenhagen, Denmark) at baseline and at 14, 16, and 19 mins during CPR and at 5, 15, 30, 60, 120, 180, and 240 mins after restoration of spontaneous circulation (ROSC). Oxygen saturation and hemoglobin were determined simultaneously on an OSM3 Hemoximeter (Radiometer). In addition, jugular vein samples were collected before cardiac arrest (baseline) and after ROSC at $5,15,30,60,120,180$, and 240 mins. The plasma obtained after centrifugation $(3500 \times$ $g$ for 12 mins) was stored at $-70^{\circ} \mathrm{C}$ until analyzed for 8-isoprostane-prostoglandin $\mathrm{F}_{2 \alpha}$ (8-iso-PGF ${ }_{2 \alpha}$ ), 15-keto-dihydro- PGF $_{2 \alpha}$, and protein S-100ß. Plasma obtained after centrif- ugation ( $4000 \times g$ for 5 mins) of arterial blood drawn at baseline and at 120 and 240 mins after ROSC was used to determine troponin I and myocardial muscle creatine kinase isoenzyme (CK-MB). Plasma glucose, lactate, and electrolyte concentrations were also determined (ABL 700, Radiometer) at baseline and 120 mins after ROSC.

Analytical Methods. Plasma concentrations of isoprostane 8-iso- $\mathrm{PGF}_{2 \alpha}$ (an indicator of oxidative injury) and 15-keto-dihydro$\mathrm{PGF}_{2 \alpha}$ (an indicator of inflammation) were measured according to a highly specific and validated radioimmunoassay method at our laboratory as previously described $(14,15)$. The detection limit for 8 -iso-PGF $\alpha$ was 23 $\mathrm{pmol} / \mathrm{L}$, and the detection limit for 15-ketodihydro- $\mathrm{PGF}_{2 \alpha}$ was $45 \mathrm{pmol} / \mathrm{L}$. Myocardial and cerebral damage were assessed by serum concentrations of cardiac troponin I, CK-MB, and astroglial protein S-100 $\beta$. Serum S- $100 \beta$ levels were measured by an immunoluminometric assay (LIA-mat, Sangtec 100, Sangtec Medical, Bromma, Sweden) with a detection limit of $0.02 \mathrm{ng} / \mathrm{mL}$ and a cutoff level of $<0.12$ ng/mL. CK-MB and troponin I were measured with monoclonal/polyclonal mass immunoassays (ADVIA Centaur, Bayer), with normal values of $<0.12 \mu \mathrm{g} / \mathrm{L}$ for troponin I and $<6 \mu \mathrm{g} / \mathrm{L}$ for CK-MB.

Experimental Protocol. After preparation, the piglets were ventilated with $30 \%$ oxygen in air and allowed to stabilize for $1 \mathrm{hr}$, after which baseline measurements were made (Table 1). Thereafter, to induce ventricular fibrillation, a $50-\mathrm{Hz}, 20-$ to $60-\mathrm{V}$ transthoracic alternating current was applied via two subcutaneous needle electrodes placed on either side of the thorax. Cardiopulmonary arrest was defined as a decrease in aortic blood pressure to hydrostatic pressure $(<25 \mathrm{~mm} \mathrm{Hg})$ and the presence of ventricular fibrillation on the electrocardiogram. Mechanical ventilation was halted at this point. After 12 mins of untreated cardiac arrest, closed-chest CPR was performed with a pneumatically driven automatic piston device (LUCAS, Jolife AB, Lund, Sweden), and mechanical ventilation with $100 \%$ oxygen was resumed with the same ventilatory settings as before induction of cardiac arrest. The animals were assigned randomly to receive an infusion of $55 \mathrm{~mL} \cdot \mathrm{kg}^{-1} \cdot \mathrm{hr}^{-1}$ saline (SAL group, $\mathrm{n}=21$ ), or $10 \mathrm{~mL} \cdot \mathrm{kg}^{-1} \cdot \mathrm{hr}^{-1}$ hypertonic saline $(7.5 \%)$ with dextran $(6 \%)$ solution (RescueFlow, Biophausia, Uppsala, Sweden) (HSD group, $\mathrm{n}=21$ ), or 7.5 $\mathrm{mg} \cdot \mathrm{kg}^{-1} \cdot \mathrm{hr}^{-1} \mathrm{MB}(10 \mathrm{mg} / \mathrm{mL}$ Metyltioninklorid, Apoteket, Umeå, Sweden) with 10 $\mathrm{mL} \cdot \mathrm{kg}^{-1} \cdot \mathrm{hr}^{-1}$ hypertonic saline-dextran solution (MB group, $n=21$ ). The dose of MB was selected after pilot experiments indicating the necessity of continuous administration with an initial loading dose (16-18) and a total dose of approximately $3 \mathrm{mg} / \mathrm{kg}$. The infusions were administered through the right atrial catheter and started after 1 min of CPR. At 1 min after commencement of CPR, all the animals received 0.4 units $/ \mathrm{kg}$ vasopressin $\left(\mathrm{Arg}^{8}{ }_{-}\right.$ 
Table 1. Experimental protocol

\begin{tabular}{|c|c|c|}
\hline Time & Intervention & Drug Administration \\
\hline & Anesthesia tracheotomy & $\begin{array}{l}\text { Xylazine, atropine, tiletamine and } \\
\text { zolazepam (Zoletil), morphine, ketamine }\end{array}$ \\
\hline & Arterial and venous cannulation & $\begin{array}{l}\text { Pentobarbital }+ \text { morphine }+ \text { pancuronium } \\
\text { bromide }\left(\mathrm{N}_{2} \mathrm{O}\right)\end{array}$ \\
\hline $1 \mathrm{hr}$ & Stabilization & $30 \%$ oxygen in air \\
\hline Baseline & Baseline parameters & \\
\hline $0 \mathrm{~min}$ & AC electric shock & Stop oxygen administration \\
\hline $0-12$ mins VF & No intervention & \\
\hline 12 mins $\mathrm{VF}$ & Start CPR & $100 \%$ oxygen \\
\hline \multirow[t]{3}{*}{13 mins VF } & & Vasopressin, 0.4 units/kg \\
\hline & Continue CPR & $\begin{array}{l}\mathrm{SAL}, 55 \mathrm{~mL} \cdot \mathrm{Kg}^{-1} \cdot \mathrm{nr}^{-1} \\
\mathrm{MB}, 7.5 \mathrm{mg} \cdot \mathrm{kg}^{-1} \cdot \mathrm{hr}^{-1}\end{array}$ \\
\hline & & $\mathrm{HSD}, 10 \mathrm{~mL} \cdot \mathrm{kg}^{-1} \cdot \mathrm{hr}^{-1}$ \\
\hline 20 mins VF & DC shock, $200 \mathrm{~J}$ & \\
\hline & Advanced life support & Epinephrine, $20 \mu \mathrm{g} / \mathrm{kg}$ \\
\hline 5 mins until ROSC & DC shock, $360 \mathrm{~J}$ & \\
\hline \multirow[t]{4}{*}{ After ROSC } & Decrease oxygen concentration & $30 \%$ oxygen in air \\
\hline & Decrease Administration of SAL, & $\mathrm{SAL}, 16.5 \mathrm{~mL} \cdot \mathrm{kg}^{-1} \cdot \mathrm{h}^{-1}$ \\
\hline & $\mathrm{HSD}, \mathrm{MB}$ & $\mathrm{MB}, 2.25 \mathrm{mg} \cdot \mathrm{kg}^{-1} \cdot \mathrm{hr}^{-1}$ \\
\hline & & $\mathrm{HSD}, 3 \mathrm{~mL} \cdot \mathrm{kg}^{-1} \cdot \mathrm{hr}^{-1}$ \\
\hline 5 mins after ROSC & Treatment of acidosis & Tris buffer mixture, $1 \mathrm{mmol} / \mathrm{kg}$ \\
\hline 50 mins & Stop infusions & Stop infusions \\
\hline 60 mins & No intervention & \\
\hline 120 mins & Monitoring & \\
\hline 180 mins & & \\
\hline 240 mins & Experiment completed & $\mathrm{KCl}, 20 \mathrm{mmol}$ \\
\hline
\end{tabular}

$\mathrm{VF}$, ventricular fibrillation; CPR, cardiopulmonary resuscitation (closed-chest CPR was performed with a pneumatically driven automatic piston device [LUCAS, Jolife AB, Lund, Sweden]); SAL, isotonic saline; MB, methylene blue; HSD, hypertonic saline (7.5\%)-dextran (6\%) solution; ROSC, restoration of spontaneous circulation.

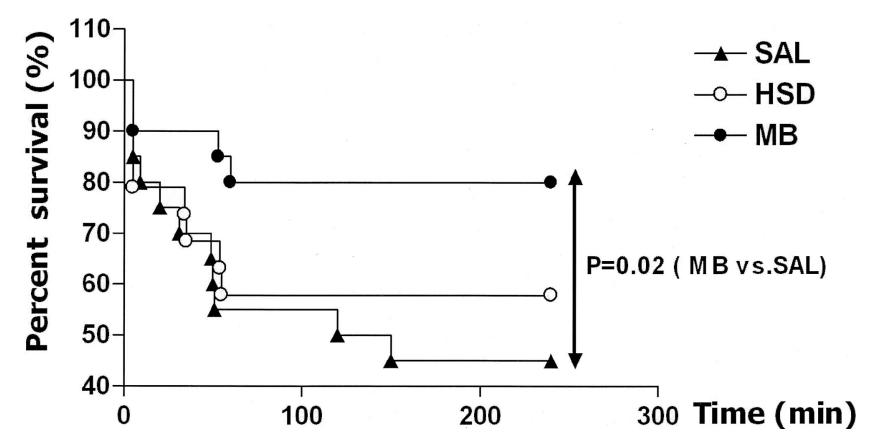

Figure 1. Kaplan-Maier survival curve indicating significant difference by the log-rank test in survival between the methylene blue and hypertonic saline with dextran group $(M B)$ and the isotonic saline $(S A L)$ group $(p=.02)$ and a significant difference between the three groups when tested together by the log-rank test for trend $(p=.03)$. SAL group, $\mathrm{n}=20$; hypertonic saline-dextran-only group $(H S D)$, $\mathrm{n}=19$; MB group, $\mathrm{n}=20$.

vasopressin) as a bolus administered via the right atrial catheter. After 8 mins of external chest compressions, a monophasic countershock was delivered through defibrillation electrode pads (Medtronic Physio-Control, Seattle, WA) at an energy level of $200 \mathrm{~J}$ (Table 1). If ROSC was not accomplished, another two defibrillatory shocks $(200 \mathrm{~J}, 360 \mathrm{~J})$ and a bolus injection of epinephrine $(20 \mu \mathrm{g} / \mathrm{kg})$ were administered and CPR continued. Direct current shocks were then applied at the energy level of $360 \mathrm{~J}$ during a maximum period of 5 mins. CPR was discontinued if ROSC was not achieved during this time. ROSC was defined as return of coordinated electrical activity resulting in a systolic blood pressure of $>60 \mathrm{~mm} \mathrm{Hg}$ for at least ten consecutive minutes. After ROSC, the infusions were reduced as follows to: 16.5 $\mathrm{mL} \cdot \mathrm{kg}^{-1} \cdot \mathrm{hr}^{-1}$ (saline), $3 \mathrm{~mL} \cdot \mathrm{kg}^{-1} \cdot \mathrm{hr}^{-1}$ (hypertonic saline-dextran), and $2.25 \mathrm{mg} \cdot \mathrm{kg}^{-1} \cdot \mathrm{hr}^{-1}$ (MB). Infusions were continued at these rates until 50 mins after ROSC, when the infusions were stopped. After 5 mins of spontaneous circulation, $\mathrm{FIO}_{2}$ was reset at 0.3 . If arterial $\mathrm{pH}$ was $<7.20$ or the base deficit was $>10 \mathrm{mmol} / \mathrm{L}$ at 5 mins after ROSC, acidosis was corrected with $1 \mathrm{mmol} / \mathrm{kg}$ Tris buffer mixture (Tribonat, Kabi Fresenius, Stockholm, Sweden) and by increasing the minute ven- tilation. The target was a $\mathrm{PaCO}_{2}$ within the range of $5.0-5.5 \mathrm{kPa}$. No other intervention was undertaken during the resuscitation phase. Hemodynamic variables and blood gases were observed for 4 hrs after ROSC.

Statistical Analysis. Data are presented as mean \pm se. Repeated-measures two-way analysis of variance was used for comparisons of the groups over time after the data were shown to be normally distributed. To secure normally distributed data analysis of 8-iso$\mathrm{PGF}_{2 \alpha}$ and 15-keto-dihydro-PGF ${ }_{2 \alpha}$, analyses were done after logarithmic transformation. Where analysis of variance showed significant differences, a Bonferroni multiple comparison test was used. Survival analysis according to the hypothesis was carried out using the method of Kaplan and Meier (GraphPad PRISM version 4, GraphPad Software, San Diego, CA), and comparisons between groups were made using the log-rank test and the log-rank test for trend because survival was considered to be a variable of the ordinal type; $p$ values of $<.05$ were regarded as statistically significant.

\section{RESULTS}

A total of 63 experiments were carried out. Four of the 63 pigs were excluded from the analysis-one in each group due to severe airway infection and one in the HSD group due to technical problems during external cardiac massage. There were no significant differences between the groups in baseline measurements.

Survival. A significant difference in survival to the end of the 4-hr experimental protocol between the MB and SAL groups $(p=.02)$ was demonstrated using the Kaplan-Meier survival curve and the logrank test or the three groups tested together by the log-rank test for trend $(p=$ .03) (Fig. 1). In the MB group, ROSC was achieved in 19 of 20 animals. In the other groups, ROSC was achieved in 16 of 19 animals in the HSD group and in 17 out of 20 animals in the SAL group. A total of 16 piglets in the MB group and 12 in the HSD group survived the entire experiment, whereas in the SAL group, only nine survived. The greatest postarrest mortality occurred in the first hour in the SAL group, in which it was associated with arrhythmias, low cardiac output, and decreasing blood pressure (eight animals).

Hemodynamic Variables. After ROSC, there was a period of 5-10 mins of spontaneous overshoot in arterial and pulmonary arterial blood pressures and cardiac output in all groups $(p<.0001)$ and, thus, also in left ventricular minute work $(p<.0001)$ (Fig. 2). After this period, a hypotensive period gradually followed in all groups, with the lowest blood pressure 

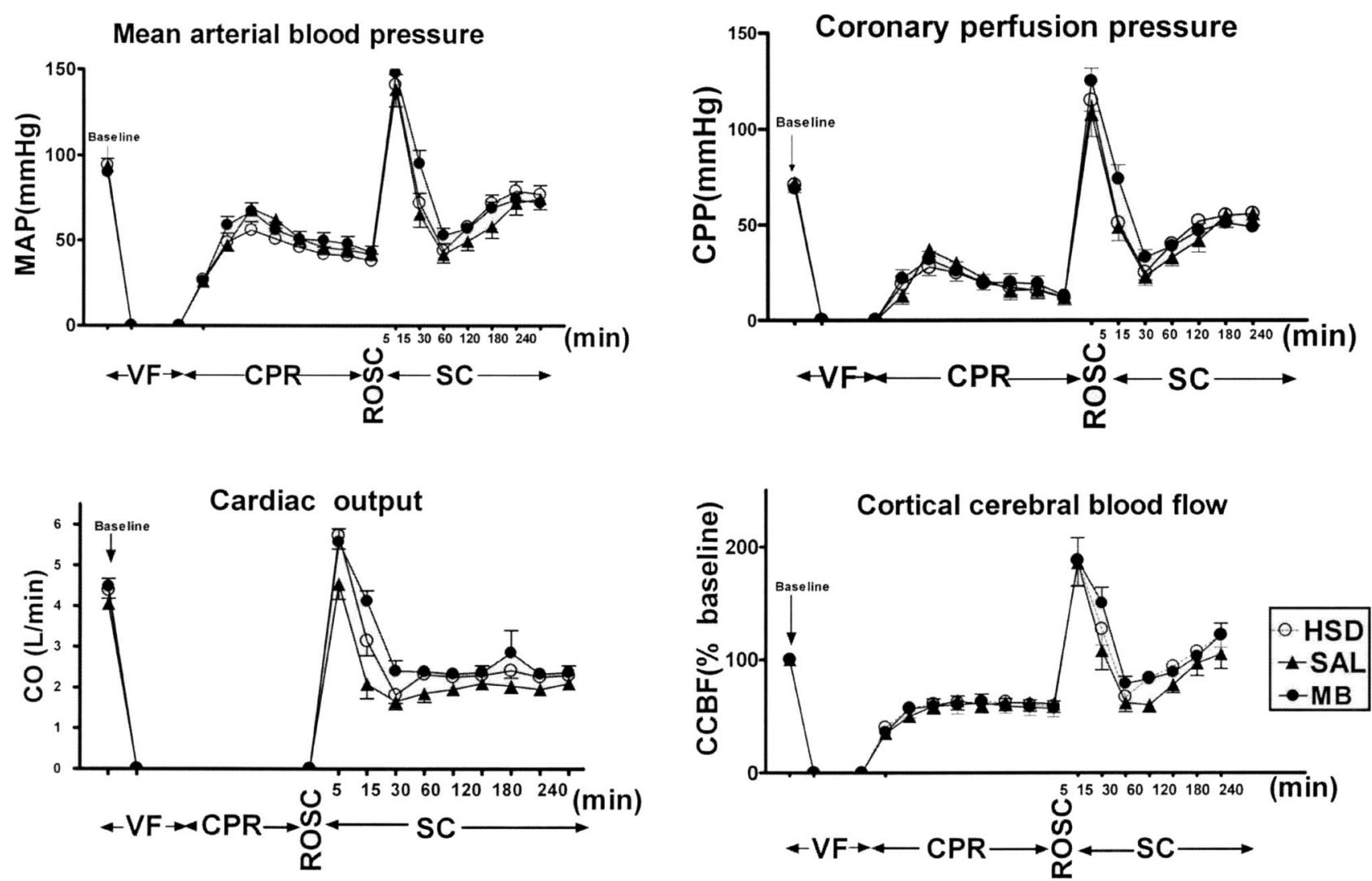

Figure 2. Five minutes after restoration of spontaneous circulation $(R O S C)$, cardiac output $(C O)$ was greater $(p<.01)$ in the methylene blue and hypertonic saline with dextran group $(M B)$ and the hypertonic saline-dextran-only group $(H S D)$ vs. the isotonic saline group $(S A L)$. There was a significantly greater mean arterial pressure $(M A P ; p<.01)$, $\mathrm{CO}(p<.05)$, and coronary perfusion pressure $(C P P ; p<.01)$ in the MB group vs. both other groups 15 mins after ROSC. During the first 60 mins after ROSC, cerebral cortical blood flow $(C C B F)$ in the MB group was greater $(p<.01)$ than that in the SAL group. Data provided as mean \pm SEM. $V F$, ventricular fibrillation; $C P R$, cardiopulmonary resuscitation; $S C$, spontaneous circulation.

levels at 30 mins compared with baseline $(p<.001)$. The decreased blood pressure lasted approximately $1 \mathrm{hr}$ after ROSC, followed by a gradual increase to baseline levels. Cardiac output was increased in both the HSD and MB groups in comparison with the SAL group $(p<.01) 5$ mins after ROSC. A greater mean arterial blood pressure $(p<.01)$, coronary perfusion pressure $(p<.01)$, cardiac output $(p<$ $.05)$, and left ventricular stroke work ( $p<$ .01 ) were observed 15 mins after ROSC in the MB group in comparison with the two other groups. The pulmonary arterial pressure was increased in the MB and HSD groups in comparison with the SAL group $(p<.05)$ at 5 and 15 mins after ROSC. In contrast, there was no significant difference between the groups in total peripheral vascular resistance.

Cerebral Cortical Blood Flow. During the very early reperfusion phase (5 mins after ROSC), the mean cerebral cortical blood flow increased in all groups in comparison with baseline values $(p<.001)$ (Fig. 2). Thereafter, this blood flow started to decrease, but there was no significant difference compared with baseline. Toward the end of the study, the cerebral cortical blood flow gradually returned to baseline in all the groups. During the first 60 mins after ROSC, the cerebral cortical blood flow differed between the groups $(p<.01)$; it was greatest in the MB group and least in the SAL group, with an intermediate value in the HSD group (not significant).

Systemic and Jugular Bulb Acid-Base Status. During the 8 mins of CPR, the arterial blood pH gradually decreased, but acidemia was not profound in any of the three groups. In contrast, acidemia was profound $(\mathrm{pH}<7.20) 5$ mins after ROSC and needed correction with Tris buffer mixture and increased ventilation. In contrast to the arterial $\mathrm{pH}$, the jugular bulb blood $\mathrm{pH}$ decreased immediately after commencement of CPR and started to increase at 16 mins after arrest.

8-iso- $P G F_{2 \alpha}$. After ROSC, the jugular bulb concentration of 8 -iso- $\mathrm{PGF}_{2 \alpha}$ increased and reached a maximum at 15 mins after ROSC (Fig. 3). Thereafter, it gradually decreased toward baseline values during the remaining part of the in- vestigation. The jugular bulb 8 -iso- $\mathrm{PGF}_{2 \alpha}$ concentration was significantly greater in the SAL group than in the MB group ( $p<$ $.01)$. There were no differences between the MB and HSD groups.

15-keto-dihydro- $P G F_{2 \alpha}$. The baseline jugular bulb concentration of 15-ketodihydro- $\mathrm{PGF}_{2 \alpha}$ increased during the early reperfusion phase immediately after ROSC (Fig. 3). Thereafter, this level decreased gradually, returning to the baseline level at the end of the study. There was a significantly lower level in the MB group in comparison with the SAL group $(p<.01)$ only.

Protein $S-100 \beta$. In all the groups, protein S-100 $\beta$ increased during the first hour after ROSC and returned to baseline levels toward the end of the study (Fig. 3). There were statistically significant differences between the three groups, with lower levels in the MB group in comparison with the SAL group $(p<.001)$ and the HSD group $(p<.01)$. There was no statistically significant difference between the SAL group and the HSD group. 

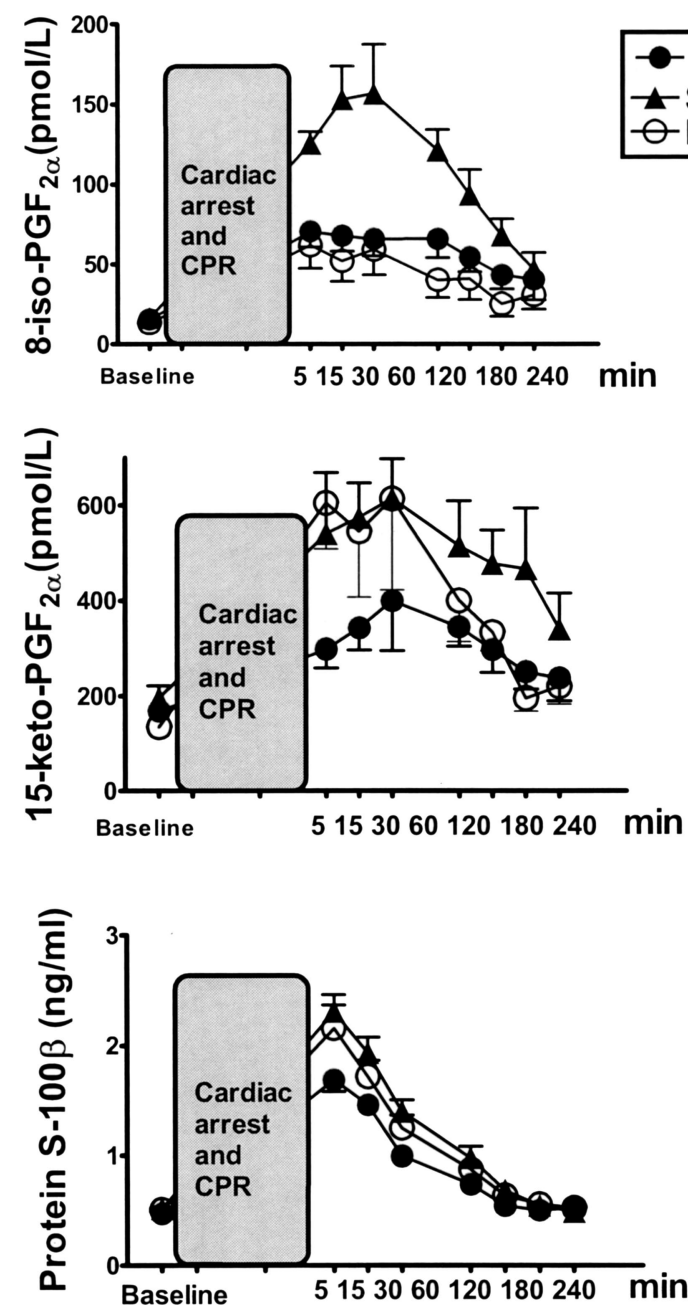

Figure 3. Jugular plasma levels of 8-isoprostane-prostaglandin $\mathrm{F}_{2 \alpha}\left(8\right.$-iso- $\left.P G F_{2 \alpha}\right)$, 15-keto-dihydro$\mathrm{PGF}_{2 \alpha}$, and protein S-100 $\beta$ at baseline and after restoration of spontaneous circulation (ROSC). After ROSC, the 8-iso- $\mathrm{PGF}_{2 \alpha}$ and 15-keto- $\mathrm{PGF}_{2 \alpha}$ levels in the methylene blue and hypertonic saline with dextran group $(M B)$ were significantly lower than in the isotonic saline group $(S A L ; p<.01)$, whereas the protein S-100 $\beta$ level in the MB group was lower in comparison with the SAL group $(p<.001)$ and the hypertonic saline-dextran-only group $(H S D ; p<.01)$. Data provided as mean \pm SEM. SAL group, $\mathrm{n}=20$; HSD group, $\mathrm{n}=19$; $\mathrm{MB}$ group, $\mathrm{n}=20$. $C P R$, cardiopulmonary resuscitation.

Myocardial Injury. Both biochemical markers of myocardial injury increased during the whole experiment, with maximums toward the end of the experiment (Fig. 4). Statistically significant differences occurred between troponin I levels in the MB and SAL groups at 240 mins $(p<.01)$. There were also statistically significant differences between CK-MB levels in the MB and SAL groups at 120 and 240 mins $(p<.05$ and $p<.01$, respectively). In contrast, there were no statistically significant differences between the HSD and SAL groups.

Lactate. Plasma lactate levels increased during the experiment in all three groups (significant differences in all the groups at 120 mins in comparison with baseline, $p<.0001)$. There was a significantly lower lactate level in the MB group compared with the SAL group at 120 mins $(p<.01)$. There was no difference between the HSD and MB groups at this time point.

\section{DISCUSSION}

Survival. The present experimental cardiac arrest study revealed that continuously administered MB and hypertonic saline with dextran during CPR, and continued for 50 mins after ROSC, increased short-term (4-hr) survival in comparison with the group receiving normal saline during the course of this experiment. In addition, the group that received MB (MB group) showed less hypotension and better cardiac performance and coronary perfusion pressure initially after return of spontaneous circulation and showed fewer signs of cerebral and cardiac injury.

Previous studies have shown that nonselective inhibitors of NOS, such as $\mathrm{N}^{\mathrm{G}}$ nitro-L-arginine methyl ester and $\mathrm{N}^{\mathrm{G}}$ monomethyl-L-arginine, reversed systemic vasodilatation and improved hemodynamics $(19,20)$. By $\mathrm{N}^{\mathrm{G}}$-nitro-L-arginine methyl ester blockage of NOS, perfusion pressure has been demonstrated to be enhanced in a cardiac arrest pig model, which resulted in a dramatic improvement in short-term survival in treated pigs compared with those receiving placebo (3). NOS inhibition has been claimed to be beneficial during CPR and reperfusion by causing an increase in systemic vascular resistance, attenuation of cerebral hyperemia, and by acting as a neuroprotective agent (21). However, whether NOS inhibition is beneficial or detrimental for hemodynamics in the postresuscitation phase is a matter of controversy $(3,21)$. In our study, the group receiving MB showed an improvement in hemodynamics in the first hour after ROSC in comparison with the SAL group. However, it must be noted that in comparison with the above-mentioned NOS inhibitors, MB exerts a mixture of effects, and NOS inhibition is only one of them $(5-9,22)$. The MB group had a significantly smaller serum lactate concentration 2 hrs after ROSC, which may indicate improved tissue perfusion in addition to its redox effects (see below) and is in accordance with the increased rate of survival in that group (23).

Myocardial Protection and Hemodynamics. Our data confirmed that MB given concomitantly with the hypertonic-hyperoncotic solution improved postresuscitation myocardial dysfunction, resulting in an increasing arterial blood pressure and cardiac output without increasing cardiac ischemia and without any change in pulse rate, total peripheral resistance, or pulmonary artery occlusion pressure. The following mechanisms could be responsible for the observed beneficial effects of this combination of events:

1) An improvement was observed in coronary perfusion pressure, initially after ROSC, simultaneously with an increase in cardiac stroke work but without an increase in total peripheral resistance. It has previously been demonstrated that nonselective inhibition of $\mathrm{NO}$ by $\mathrm{N}^{\mathrm{G}}$-monomethyl-Larginine increases coronary blood flow after ischemia, thus improving 


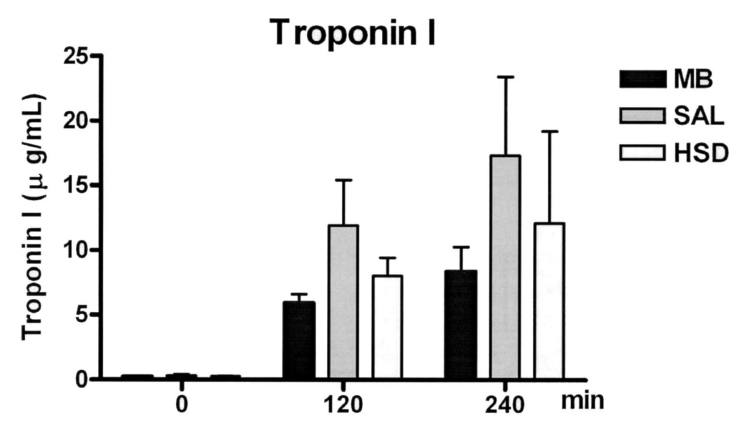

CKMB

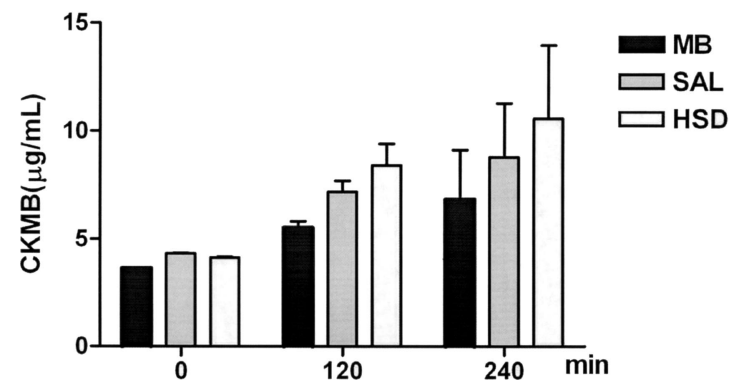

Figure 4. Systemic plasma levels of troponin I and myocardial muscle creatine kinase isoenzyme $(C K M B)$ at baseline and after restoration of spontaneous circulation $(R O S C)$. The troponin I level in the isotonic saline group $(S A L)$ was greater than in that the methylene blue and hypertonic saline with dextran group $(M B)$ at 240 mins $(p<.01)$. The CKMB level in the SAL group was greater than that in the MB group at 120 mins $(p<.05)$ and 240 mins $(p<.01)$, respectively. Data provided as mean \pm SEM. SAL group, $\mathrm{n}=20$; hypertonic saline-dextran-only group (HSD), $\mathrm{n}=19$; $\mathrm{MB}$ group, $\mathrm{n}=20$.

contractility (24). NO affects myocardial contraction in a dose-dependent fashion, with low doses of $\mathrm{NO}$ resulting in positive inotropic effects and higher concentrations exerting negative inotropic effects (25). Resuscitation studies using hypertonic-hyperoncotic solutions have usually reported an improvement in myocardial blood flow during CPR (11) as a result of improved microcirculation during ischemia (26), whereas only minor effects were found during recirculation $(26,27)$. In our investigation, significant statistical, but rather small and of relatively short duration, differences in mean arterial blood pressure, coronary perfusion pressure, and left ventricular stroke work occurred not only between the MB and SAL groups at 5-15 mins after ROSC, but also between the MB and HSD groups. Thus, these results could not be related solely to the administration of HSD solution.

2) MB blocks the formation of several reactive oxygen species, particularly superoxides, by competing with molecular oxygen for the transfer of electrons by xanthine oxidase (8). Per- oxynitrite $\left(\cdot \mathrm{ONOO}^{-1}\right)$, a product of $\mathrm{NO}$ and superoxide anion $\left(\cdot \mathrm{O}_{2}\right)$ interaction, is known as a powerful oxidant exerting vasorelaxant effects on peripheral blood vessels (28). Peroxynitrite depresses myocardial contractility by decreasing the ability of $\mathrm{Ca}^{2+}$ to trigger contraction, an effect that is partly mediated by the cGMP/cGMP-dependent protein kinase pathway (29). The presence of MB significantly inhibits the relaxation induced by peroxynitrite (30), and it promotes myocardial contraction (31).

Previous studies have demonstrated that hypertonic-hyperoncotic solutions reduce the release of cardiac troponin I after successful CPR, even in the absence of the hemodynamic improvement (12). The levels of troponin I and CK-MB continuously increased throughout the present experiment, and the results reflect a favorable effect of MB in myocardial injury, with lower levels of CK-MB and troponin I in the MB group, despite the greater left ventricular stroke work early after ROSC in this group. In addition, the systemic plasma level of lactate was significantly lower in the MB group compared with the SAL group. This effect of MB has previously been described (32) and considered to be a re-oxidizing effect (by leuco-MB) of nicotinamide adenine dinucleotide phosphate leading to a reduction of the lactate/pyruvate ratio.

Cerebral Protection. Induction of regional cerebral hyperemia has been suggested as the mechanism linking regional neuronal activity with regional cerebral blood flow (33). NO, a potent vasodilator released during synaptic activity, has emerged as an important factor in the development of functional hyperemia (33, 34). Systemic hypertension that commonly occurs immediately after successful resuscitation also results in increased cerebral blood flow. This hyperemia has been suggested as a major contributor to damage to the blood-brain barrier (35). Indeed, the greatest signs of lipid peroxidation after CPR were previously recorded in one of our experimental study groups (36) in which hyperemia was greatest and autoregulation of cerebral blood flow was least. Thus, attenuation of cerebral hyperemia and promotion of circulatory autoregulation might improve outcome after CPR. The use of a nonspecific NOS inhibitor, $\mathrm{N}^{\mathrm{G}}$-nitro-Larginine methyl ester, at a relatively low dose attenuated the early hyperemic response to ischemia-reperfusion in certain brain regions (21), most notably the brain stem, resulting in an overall reduction in cerebral blood flow. However, in the present investigation, no differences in cerebral cortical blood flow during CPR or soon after ROSC were found between the groups, in which a mild, statistically nonsignificant cerebral hypoperfusion occurred in all three groups of animals during the period from 15 mins to $2 \mathrm{hrs}$ after ROSC.

The astroglial protein S- $100 \beta$ is a sensitive marker of hypoxic brain damage after cardiac arrest, and it correlates well with the extent and prognosis of ischemic brain injury $(37,38)$. Protein S- $100 \beta$ also reflects the extent of experimental brain damage after cerebral ischemia and serves as a useful biomarker for the assessment of effects of neuroprotective drugs $(37,38)$. In the present study, we found that the levels of protein S-100ß were significantly lower during the course of our experiments in the MB group, indicating neuroprotective effect in this group, as in a previous study demonstrating that MB acts as a neuroprotective agent by inhibiting brain NOS activity in vivo and interfering with the NO-cGMP system (39). In ischemia-reperfusion, neuronal NOS activity is increased 
and the excess of subsequently liberated NO becomes neurotoxic (40). A possible mechanism by which neurotoxicity is induced is the formation of peroxynitrite anion (40, 41). Thus, NOS inhibitors are considered to favor neuroprotection by inhibiting NO production, preventing neurotoxicity due to excess $\mathrm{NO}$ by scavenging oxygen-derived free radicals $(41,42)$, which together may otherwise form peroxynitrite.

Successful resuscitation is associated not only with cellular brain damage (necrosis and apoptosis) but also with local and systemic inflammatory response. Jugular bulb isoprostanes and $\mathrm{PGF}_{2 \alpha}$ metabolites have previously been proved to serve as biomarkers of oxidative free-radical damage and inflammatory response in the brain after reperfusion injury (43, 44). Isoprostanes are biosynthesized from arachidonic acid in vivo, mainly through nonenzymatic free-radical-catalyzed oxidation. Prolonged duration of cardiac arrest and CPR result in a worsening of the oxidative injury to cerebral tissue (45) and subsequent neurologic damage. The levels of 8-iso- $\mathrm{PGF}_{2 \alpha}$ were significantly reduced in the postresuscitation phase in the MB group in comparison with the SAL group, indicating less oxidative injury, but no difference was recorded in relation to the HSD group.

NO enhances cyclooxygenase activity through a mechanism independent of cGMP, and in conditions in which both NOS and cyclooxygenase are activated, there is an NO-mediated increase in the production of proinflammatory prostaglandins, which may result in exacerbated inflammatory response (46). Enzymatic catalysis by cyclooxygenases, leading to formation of prostaglandins from arachidonic acid and their involvement in the inflammatory process and 15-keto-dihydro- $\mathrm{PGF}_{2 \alpha}$, is increased in inflammation and can be used as an indicator of in vivo lipid oxidation through the cyclooxygenase pathway (47). MB inhibits the metabolism of arachidonic acid in both the cyclooxygenase and lipoxygenase pathways (48). This interaction explains the results obtained in the present study, in which 15-keto-dyhydro$\mathrm{PGF}_{2 \alpha}$ was significantly decreased in the MB group in comparison with both the other groups. Thus, MB decreased the cerebral inflammatory response after reperfusion injury.

\section{CONCLUSION}

MB added to a hypertonic-hyperoncotic solution increased short-term (4- hr) survival after cardiac arrest and CPR as compared with a control group given normal saline only and acted as a cardioand cerebro-protective pharmacologic agent. Further studies should be carried out to define the role of MB in the treatment of cardiac arrest.

\section{REFERENCES}

1. Xu XP, Pollock JS, Tanner MA, et al: Hypoxia activates nitric oxide synthase and stimulates nitric oxide production in porcine coronary resistance arteriolar endothelial cells. Cardiovasc Res 1995; 30:841-847

2. Furchgott RF, Jothianandan D: Endotheliumdependent and -independent vasodilation involving cyclic GMP: Relaxation induced by nitric oxide, carbon monoxide and light. Blood Vessels 1991; 28:52-61

3. Krismer AC, Lindner $\mathrm{KH}$, Wenzel $\mathrm{V}$, et al: Inhibition of nitric oxide improves coronary perfusion pressure and return of spontaneous circulation in a porcine cardiopulmonary resuscitation model. Crit Care Med 2001; 29: 482-486

4. Miki N, Kawabe Y, Kuriyama K: Activation of cerebral guanylate cyclase by nitric oxide. Biochem Biophys Res Commun 1977; 75: 851-856

5. Martin W, Villani GM, Jothianandan D, et al: Selective blockade of endothelium-dependent and glyceryl trinitrate-induced relaxation by hemoglobin and by methylene blue in the rabbit aorta. J Pharmacol Exp Ther 1985; 232:708-716

6. Tsai SC, Adamik R, Manganiello VC, et al: Regulation of activity of purified guanylate cyclase from liver that is unresponsive to nitric oxide. Biochem J 1983; 215:447-455

7. Wolin MS, Cherry PD, Rodenburg JM, et al: Methylene blue inhibits vasodilation of skeletal muscle arterioles to acetylcholine and nitric oxide via the extracellular generation of superoxide anion. J Pharmacol Exp Ther 1990; 254:872-876

8. Salaris SC, Babbs CF, Voorhees WD III: Methylene blue as an inhibitor of superoxide generation by xanthine oxidase: A potential new drug for the attenuation of ischemia/ reperfusion injury. Biochem Pharmacol 1991; 42:499-506

9. Kelner MJ, Bagnell R, Hale B, et al: Potential of methylene blue to block oxygen radical generation in reperfusion injury. Basic Life Sci 1988; 49:895-898

10. Negovsky V, Gurvitch A, Zolotokrylina E: Post-resuscitation Disease. Amsterdam, Elsevier, 1983

11. Breil M, Krep H, Sinn D, et al: Hypertonic saline improves myocardial blood flow during CPR, but is not enhanced further by the addition of hydroxy ethyl starch. Resuscitation 2003; 56:307-317

12. Krieter H, Denz C, Janke C, et al: Hypertonichyperoncotic solutions reduce the release of cardiac troponin I and s-100 after successful cardiopulmonary resuscitation in pigs. Anesth Analg 2002; 95:1031-1036

13. Bertsch T, Denz C, Janke C, et al: Hypertonichyperoncotic solutions decrease cardiac troponin I concentrations in peripheral blood in a porcine ischemia-reperfusion model. Exp Toxicol Pathol 2001; 53:153-156

14. Basu S: Radioimmunoassay of 8-iso-prostaglandin F2alpha: An index for oxidative injury via free radical catalysed lipid peroxidation. Prostaglandins Leukot Essent Fatty Acids 1998; 58:319-325

15. Basu S: Radioimmunoassay of 15-keto-13,14dihydro-prostaglandin F2alpha: An index for inflammation via cyclooxygenase catalysed lipid peroxidation. Prostaglandins Leukot Essent Fatty Acids 1998; 58:347-352

16. Burrows GE: Methylene blue: Effects and disposition in sheep. $J$ Vet Pharmacol Ther 1984; 7:225-231

17. Basu S, Nozari A, Liu XL, et al: Development of a novel biomarker of free radical damage in reperfusion injury after cardiac arrest. FEBS Lett 2000; 470:1-6

18. Blass N, Fung D: Dyed but not dead-methylene blue overdose. Anesthesiology 1976; 45: 458-459

19. Hua TC, Moochhala SM: Influence of Larginine, aminoguanidine, and NG-nitro-Larginine methyl ester (L-name) on the survival rate in a rat model of hemorrhagic shock. Shock 1999; 11:51-57

20. Cotter G, Kaluski E, Blatt A, et al: L-NMMA (a nitric oxide synthase inhibitor) is effective in the treatment of cardiogenic shock. Circulation 2000; 101:1358-1361

21. Schleien CL, Kuluz JW, Gelman B: Hemodynamic effects of nitric oxide synthase inhibition before and after cardiac arrest in infant piglets. Am J Physiol 1998; 274:H1378-H1385

22. Mayer B, Brunner F, Schmidt K: Inhibition of nitric oxide synthesis by methylene blue. Biochem Pharmacol 1993; 45:367-374

23. Mullner M, Sterz F, Domanovits H, et al: The association between blood lactate concentration on admission, duration of cardiac arrest, and functional neurological recovery in patients resuscitated from ventricular fibrillation. Intensive Care Med 1997; 23:1138-1143

24. Parrino PE, Laubach VE, Gaughen JR Jr, et al: Inhibition of inducible nitric oxide synthase after myocardial ischemia increases coronary flow. Ann Thorac Surg 1998; 66: 733-739

25. Mohan P, Brutsaert DL, Paulus WJ, et al: Myocardial contractile response to nitric oxide and cGMP. Circulation 1996; 93: 1223-1229

26. Fischer M, Dahmen A, Standop J, et al: Effects of hypertonic saline on myocardial blood flow in a porcine model of prolonged cardiac arrest. Resuscitation 2002; 54:269-280

27. Nozari A, Rubertsson S, Gedeborg R, et al: Maximisation of cerebral blood flow during experimental cardiopulmonary resuscitation does not ameliorate post-resuscitation hypoperfusion. Resuscitation 1999; 40:27-35

28. Beckman JS, Beckman TW, Chen J, et al: 
Apparent hydroxyl radical production by peroxynitrite: Implications for endothelial injury from nitric oxide and superoxide. Proc Nat Acad Sci U S A 1990; 87:1620-1624

29. Brunner F, Wolkart G: Peroxynitrite-induced cardiac depression: Role of myofilament desensitization and cGMP pathway. Cardiovasc Res 2003; 60:355-364

30. Li J, Li W, Altura BT, et al: Peroxynitriteinduced relaxation in isolated rat aortic rings and mechanisms of action. Toxicol Appl Pharmacol 2005; 209:269-276

31. Levin RL, Degrange MA, Bruno GF, et al: Methylene blue reduces mortality and morbidity in vasoplegic patients after cardiac surgery. Ann Thorac Surg 2004; 77:496-499

32. Tranquada R, Bernstein S, Grant W: Methylene blue in the treatment of lactic acidosis. Clin Res 1963; 11:230-235

33. Yang G, Zhang $\mathrm{Y}$, Ross ME, et al: Attenuation of activity-induced increases in cerebellar blood flow in mice lacking neuronal nitric oxide synthase. Am J Physiol Heart Circ Physiol 2003; 285:H298-H304

34. Gally JA, Montague PR, Reeke GN Jr, et al: The NO hypothesis: possible effects of a short-lived, rapidly diffusible signal in the development and function of the nervous system. Proc Nat Acad Sci U S A 1990; 87: 3547-3551
35. Schleien CL, Koehler RC, Shaffner DH, et al: Blood-brain barrier disruption after cardiopulmonary resuscitation in immature swine. Stroke 1991; 22:477-483

36. Liu XL, Wiklund L, Nozari A, et al: Differences in cerebral reperfusion and oxidative injury after cardiac arrest in pigs. Acta Anaesthesiol Scand 2003; 47:958-967

37. Shirasaki Y, Edo N, Sato T: Serum S-100b protein as a biomarker for the assessment of neuroprotectants. Brain Res 2004; 1021: 159-166

38. Bottiger BW, Mobes S, Glatzer R, et al: Astroglial protein S-100 is an early and sensitive marker of hypoxic brain damage and outcome after cardiac arrest in humans. Circulation 2001; 103:2694-2698

39. Volke V, Wegener G, Vasar E, et al: Methylene blue inhibits hippocampal nitric oxide synthase activity in vivo. Brain Res 1999; 826: 303-305

40. Bolanos JP, Almeida A: Roles of nitric oxide in brain hypoxia-ischemia. Biochim Biophys Acta 1999; 1411:415-436

41. Margaill I, Allix M, Boulu RG, et al: Dose- and time-dependence of L-NAME neuroprotection in transient focal cerebral ischaemia in rats. Br J Pharmacol 1997; 120:160-163

42. Beckman JS, Ye YZ, Chen J, et al: The interactions of nitric oxide with oxygen radicals and scavengers in cerebral ischemic injury. Adv Neurol 1996; 71:339-350

43. Liu XL, Nozari A, Basu S, et al: Neurological outcome after experimental cardiopulmonary resuscitation: A result of delayed and potentially treatable neuronal injury? Acta Anaesthesiol Scand 2002; 46:537-546

44. Idris AH, Roberts LJ Jr, Caruso L, et al: Oxidant injury occurs rapidly after cardiac arrest, cardiopulmonary resuscitation, and reperfusion. Crit Care Med 2005; 33:2043-2048

45. Basu S, Liu X, Nozari A, et al: Evidence for time-dependent maximum increase of free radical damage and eicosanoid formation in the brain as related to duration of cardiac arrest and cardio-pulmonary resuscitation. Free Radic Res 2003; 37:251-256

46. Salvemini D, Misko TP, Masferrer JL, et al: Nitric oxide activates cyclooxygenase enzymes. Proc Nat Acad Sci U S A 1993; 90: $7240-7244$

47. Vane J, Botting R: A better understanding of anti-inflammatory drugs based on isoforms of cyclooxygenase (COX-1 and COX-2). Adv Prostaglandin Thromboxane Leukot Res 1995; 23:41-48

48. Losche W, Bosia A, Heller R, et al: Methylene blue inhibits the arachidonic acid metabolism in human blood platelets. Biomed Biochim Acta 1988; 47:S100-S103 